\title{
Ringing of the Regular Black Hole with Asymptotically Minkowski Core
}

\author{
Alexander Marcus Simpson \\ School of Mathematics and Statistics, Victoria University of Wellington, P.O. Box 600, \\ Wellington 6140, New Zealand; alex.simpson@sms.vuw.ac.nz
}

check for updates

Citation: Simpson, A.M. Ringing of the Regular Black Hole with Asymptotically Minkowski Core. Universe 2021, 7, 418. https:// doi.org/10.3390/universe7110418

Academic Editors: Hiroyuki Nakano and Nami Uchikata

Received: 24 September 2021

Accepted: 1 November 2021

Published: 2 November 2021

Publisher's Note: MDPI stays neutral with regard to jurisdictional claims in published maps and institutional affiliations.

Copyright: (C) 2021 by the author. Licensee MDPI, Basel, Switzerland. This article is an open access article distributed under the terms and conditions of the Creative Commons Attribution (CC BY) license (https:/ / creativecommons.org/licenses/by/ $4.0 /)$.
Abstract: A Regge-Wheeler analysis is performed for a novel black hole mimicker 'the regular black hole with asymptotically Minkowski core', followed by an approximation of the permitted quasi-normal modes for propagating waveforms. A first-order WKB approximation is computed for spin zero and spin one perturbations of the candidate spacetime. Subsequently, numerical results analysing the respective fundamental modes are compiled for various values of the $a$ parameter (which quantifies the distortion from Schwarzschild spacetime), and for various multipole numbers $\ell$. Both electromagnetic spin one fluctuations and scalar spin zero fluctuations on the background spacetime are found to possess shorter-lived, higher-energy signals than their Schwarzschild counterparts for a specific range of interesting values of the $a$ parameter. Comparison between these results and some analogous results for both the Bardeen and Hayward regular black holes is considered. Analysis as to what happens when one permits perturbations of the Regge-Wheeler potential itself is then conducted, first in full generality, before specialising to Schwarzschild spacetime. A general result is presented explicating the shift in quasi-normal modes under perturbation of the Regge-Wheeler potential.

Keywords: regular black hole; Minkowski core; Lambert $W$ function; black hole mimic; ReggeWheeler potential; quasi-normal modes; WKB approximation

\section{Introduction}

Given the conditions that a propagating waveform is purely ingoing at the horizon and purely outgoing at spatial infinity, the proper oscillation frequencies of a candidate black hole spacetime are determined via analysis of the permitted quasi-normal modes (QNMs). QNM analysis is by now utterly standard, with a wealth of literature containing QNM analyses in many varied contexts [1-26], as well as the QNMs of propagating waveforms emanating from an astrophysical source being directly observed via experiment in the LIGO/VIRGO merger events [27-29]. Given the hope that LIGO/VIRGO (or more likely LISA [30]) will eventually be able to delineate the fingerprint of classical black holes from possible black hole mimickers, it is increasingly relevant to analyse well-motivated candidate spacetimes that model black hole mimickers and to compile results that speak to the advances made in observational and gravitational wave astronomy. It should be noted that such analysis is purely classical, as is consistent with the relevant ringdown calculation for LIGO/VIRGO. It is well-known that classical curvature singularities in general relativity (GR) typically occur at a distance scale that only a complete theory of quantum gravity could adequately describe. More specifically, treatments of both the classical analysis and aspects from the quantum mechanics can lead to the amelioration of curvature singularities in certain configurations [31,32]. However, in the absence of a phenomenologically falsifiable/verifiable theory of quantum gravity, it is well-motivated to construct nonsingular model spacetimes and analyse them through the lens of standard GR.

One such candidate spacetime is the regular black hole with an asymptotically Minkowski core. By 'regular black hole', one means in the sense of Bardeen [33]; a black hole with a well-defined horizon structure and everywhere-finite curvature tensors and 
curvature invariants. Regular black holes as a subject matter possess a rich genealogy; see for instance references [33-40]. For current purposes, the candidate spacetime in question is given by the line element

$$
\mathrm{d} s^{2}=-\left(1-\frac{2 m \mathrm{e}^{-a / r}}{r}\right) \mathrm{d} t^{2}+\frac{\mathrm{d} r^{2}}{1-\frac{2 m \mathrm{e}^{-a / r}}{r}}+r^{2}\left(\mathrm{~d} \theta^{2}+\sin ^{2} \theta \mathrm{d} \phi^{2}\right) .
$$

One can find thorough discussions of aspects of this specific metric in references [41,42], where causal structure, surface gravity, satisfaction/violation of the standard energy conditions, and locations of both photon spheres and timelike circular orbits are analysed through the lens of standard GR. An extremal version of this metric, and various other metrics with mathematical similarities, have also been discussed in rather different contexts [43-50].

This paper seeks to compute some of the relevant QNM profiles for this candidate spacetime. Consequently, the author first performs the necessary extraction of the specific spin-dependent Regge-Wheeler potentials in Section 2, before analysing the spin one and spin zero QNMs via the numerical technique of a first-order WKB approximation in Section 3. For specified multipole numbers $\ell$, and various values of $a$, numerical results are then compiled in Section 4. These analyse the respective fundamental modes for spin one and spin zero perturbations of a background spacetime possessing some trial astrophysical source. Brief comparison is made between these results and the analogous results for the Bardeen and Hayward regular black hole models. General perturbations of the ReggeWheeler potential itself are then analysed in Section 5, with some quite general results being presented, before concluding the discussion in Section 6.

\section{Regge-Wheeler Potential}

In this section, the spin-dependent Regge-Wheeler potentials are explored. Ultimately, the spin two axial mode involves perturbations which are somewhat messier, and hence do not lend themselves nicely to the WKB approximation and subsequent computation of quasi-normal modes without the assistance of numerical code. Due to this ensuing intractability, the relevant Regge-Wheeler potential for the spin two axial mode is explored for completeness, before specialising the QNM discourse to spin zero (scalar) and spin one (e.g., electromagnetic) perturbations only. The QNMs of spin two axial perturbations are relegated to the domain of future research. Given one does not know the spacetime dynamics a priori, the inverse Cowling approximation is invoked, where one allows the scalar/vector field of interest to oscillate while keeping the candidate geometry fixed. This formalism closely follows that of reference [51].

To proceed, one implicitly defines the tortoise coordinate via

$$
\mathrm{d} r^{*}=\frac{\mathrm{d} r}{1-\frac{2 m \mathrm{e}^{-a / r}}{r}} .
$$

Although this equation is not analytically integrable, one can still conduct analysis of the Regge-Wheeler potential through this implicit definition of the tortoise coordinate. The coordinate transformation Equation (2) allows one to write the spacetime metric Equation (1) in the following form:

$$
\mathrm{d} s^{2}=\left(1-\frac{2 m \mathrm{e}^{-a / r}}{r}\right)\left\{-\mathrm{d} t^{2}+\mathrm{d} r_{*}^{2}\right\}+r^{2}\left(\mathrm{~d} \theta^{2}+\sin ^{2} \theta \mathrm{d} \phi^{2}\right),
$$

which can then be rewritten as

$$
\mathrm{d} s^{2}=A\left(r_{*}\right)^{2}\left\{-\mathrm{d} t^{2}+\mathrm{d} r_{*}^{2}\right\}+B\left(r_{*}\right)^{2}\left(\mathrm{~d} \theta^{2}+\sin ^{2} \theta \mathrm{d} \phi^{2}\right) .
$$


In Regge and Wheeler's original work [52], they show that for perturbations in a black hole spacetime, assuming a separable wave form of the type

$$
\Psi\left(t, r_{*}, \theta, \phi\right)=\mathrm{e}^{i \omega t} \psi\left(r_{*}\right) Y(\theta, \phi)
$$

results in the following differential Equation (now called the Regge-Wheeler equation):

$$
\frac{\partial^{2} \psi\left(r_{*}\right)}{\partial r_{*}^{2}}+\left\{\omega^{2}-\mathcal{V}_{S}\right\} \psi\left(r_{*}\right)=0 .
$$

Here $Y(\theta, \phi)$ represents the spherical harmonic functions, $\psi\left(r_{*}\right)$ is a propagating scalar, vector, or spin two axial bivector field in the candidate spacetime, $\mathcal{V}_{S}$ is the spin-dependent Regge-Wheeler potential, and $\omega$ is some (possibly complex) temporal frequency in the Fourier domain $[15,22,23,38,51-53]$. The method for solving Equation (6) is dependent on the spin of the perturbations and on the background spacetime. For instance, for vector perturbations $(S=1)$, specialising to electromagnetic fluctuations, one analyses the electromagnetic four-potential subject to Maxwell's equations:

$$
\frac{1}{\sqrt{-g}} \partial_{\mu}\left(F^{\mu v} \sqrt{-g}\right)=0
$$

while for scalar perturbations $(S=0)$, one solves the minimally coupled massless KleinGordon equation

$$
\square \psi(r)=\frac{1}{\sqrt{-g}} \partial_{\mu}\left(\sqrt{-g} \partial^{\mu} \psi\right)=0 .
$$

Further details can be found in references [23,24,51,52]. For spins $S \in\{0,1,2\}$, this yields the general result in static spherical symmetry [51,53]:

$$
\mathcal{V}_{0,1,2}=\left\{\frac{A^{2}}{B^{2}}\right\}\left[\ell(\ell+1)+S(S-1)\left(g^{r r}-1\right)\right]+(1-S) \frac{\partial_{r_{*}}^{2} B}{B},
$$

where $A$ and $B$ are the relevant functions as specified by Equation (4), $\ell$ is the multipole number (with $\ell \geq S$ ), and $g^{r r}$ is the relevant contrametric component with respect to standard curvature coordinates (for which the covariant components are presented in Equation (1)).

For the spacetime under consideration, one has $A(r)=\sqrt{1-\frac{2 m \mathrm{e}^{-a / r}}{r}}, B(r)=r$, $g^{r r}=1-\frac{2 m \mathrm{e}^{-a / r}}{r}$, and $\partial_{r_{*}}=\left(1-\frac{2 m \mathrm{e}^{-a / r}}{r}\right) \partial_{r}$. Hence,

$$
\frac{\partial_{r_{*}}^{2} B}{B}=\frac{\left(1-\frac{2 m \mathrm{e}^{-a / r}}{r}\right) \partial_{r}\left[1-\frac{2 m \mathrm{e}^{-a / r}}{r}\right]}{r}=\left(\frac{r-2 m \mathrm{e}^{-a / r}}{r^{3}}\right)\left(\frac{2 m \mathrm{e}^{-a / r}(r-a)}{r^{2}}\right),
$$

and so one has the exact result that

$$
\mathcal{V}_{0,1,2}=\left(\frac{r-2 m \mathrm{e}^{-a / r}}{r^{3}}\right)\left\{\ell(\ell+1)+\frac{2 m \mathrm{e}^{-a / r}}{r}(1-S)\left[S+1-\frac{a}{r}\right]\right\} .
$$

That is,

$$
\mathcal{V}_{0,1,2}=\left(1-\frac{2 m \mathrm{e}^{-a / r}}{r}\right)\left\{\frac{\ell(\ell+1)}{r^{2}}+\frac{2 m \mathrm{e}^{-a / r}}{r^{3}}(1-S)\left[S+1-\frac{a}{r}\right]\right\} .
$$

Please note that at the outer horizon, $r_{H}=2 m \mathrm{e}^{\mathrm{W}\left(-\frac{a}{2 m}\right)}$, with $W$ being the special Lambert $W$ function [51,53-64], the Regge-Wheeler potential vanishes. Taking the limit as 
$a \rightarrow 0$ recovers the known Regge-Wheeler potentials for spin zero, spin one, and spin two axial perturbations in the Schwarzschild spacetime:

$$
\mathcal{V}_{S c h ., 0,1,2}=\lim _{a \rightarrow 0} \mathcal{V}_{0,1,2}=\left(1-\frac{2 m}{r}\right)\left\{\frac{\ell(\ell+1)}{r^{2}}+\frac{2 m}{r^{3}}\left(1-S^{2}\right)\right\} .
$$

Please note that in Regge and Wheeler's original work [52], only the spin two axial mode was analysed. However, this result agrees both with the original work, as well as with later results extending to spin zero and spin one perturbations [23]. It is informative to explicate the exact form for the RW-potential for each spin case, and to then plot the qualitative behaviour of the potential as a function of the dimensionless variables $r / m$ and $a / m$ for the respective dominant multipole numbers $(\ell=S)$.

- Spin one vector field: The conformal invariance of spin one massless particles in $(3+1)$ dimensions implies that the $\frac{\partial_{r_{*}}^{2} B}{B}$ term vanishes, and indeed mathematically the potential reduces to the highly tractable

$$
\mathcal{V}_{1}=\left(1-\frac{2 m \mathrm{e}^{-a / r}}{r}\right) \frac{\ell(\ell+1)}{r^{2}}
$$

Specialising to the dominant multipole number $\ell=1$ gives:

$$
\left.\mathcal{V}_{1}\right|_{\ell=1}=\frac{2}{r^{2}}\left(1-\frac{2 m \mathrm{e}^{-a / r}}{r}\right) .
$$

Now, in order to examine the qualitative features of the potential it is of mathematical convenience to define the new dimensionless variables $x=r / m$, and $y=a / m$. It is worth noting here that convention in the historical literature would be to set $a \sim m_{p}$, such that the newly introduced scalar parameter appeals to the quantum gravity regime. This would imply that $y=a / m \sim m_{p} / m_{\text {sun }} \ll 1$. In view of the redefinition of parameters, Equation (15) may be re-expressed as follows:

$$
\left.m^{2} \mathcal{V}_{1}\right|_{\ell=1}=\frac{2}{x^{2}}\left(1-\frac{2 \mathrm{e}^{-y / x}}{x}\right)
$$

The qualitative features of $\mathcal{V}_{1}$ are then plotted in Figure 1, for the full range of $y$ such that the spacetime still possesses a nontrivial horizon structure, and the domain for $x$ such that one is strictly outside the horizon.

An immediate sanity check from Figure 1 is that for $a=0$, where the candidate spacetime reduces to Schwarzschild, one observes a peak at $r=3 m$. This is the expected location of the photon sphere for Schwarzschild, and is indeed the corresponding location of the peak of the relevant spin one RW-potential. As $a$ increases, the $r$-coordinate location of the peak decreases. For all values of $a$, there is falloff at spatial infinity, and once the peak is crested there is rapid falloff as one approaches the horizon location (where the RW-potential vanishes completely). The green line present in Figure $1 \mathrm{~b}$ corresponds to the approximate location of the photon sphere calculated in reference [42]; $r_{\gamma} \approx 3 m-\frac{4}{3} a$. This approximation is used for the location of the peak of the spin one potential in order to extract the QNM profile approximations in Section 3. One can see that for the given domain and range this approximation has high accuracy, closely matching with the locations of the peaks. 

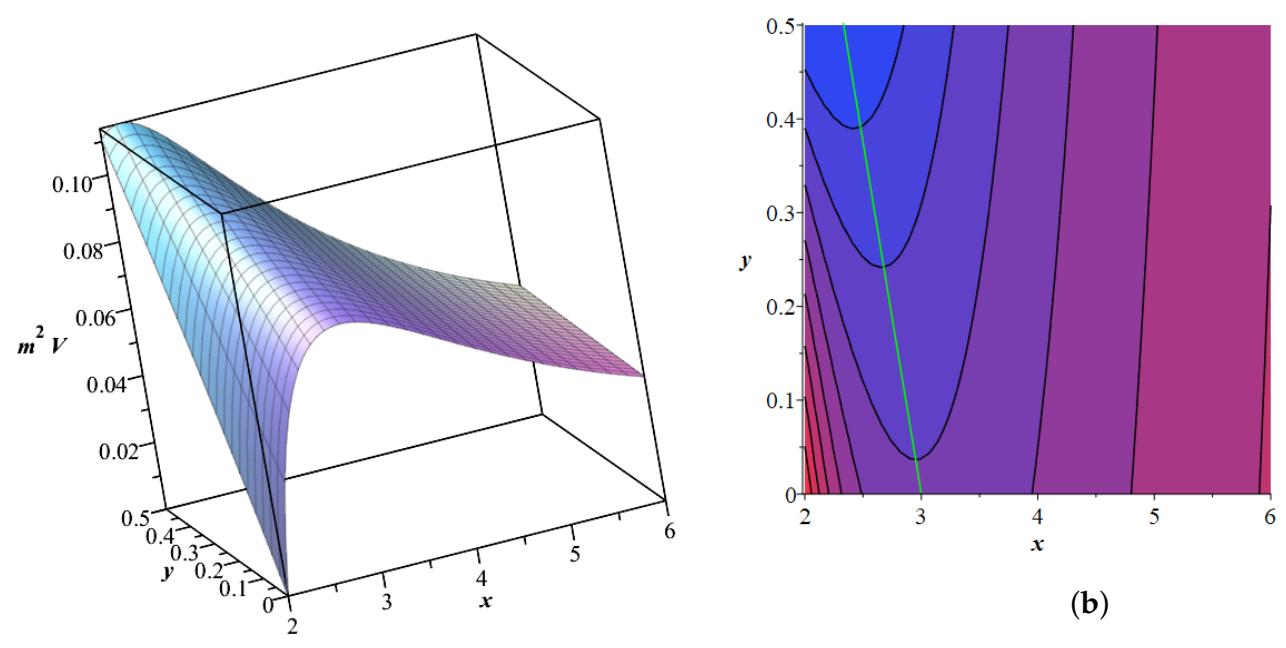

(a)

Figure 1. The qualitative features of the spin one Regge-Wheeler potential for the dominant multipole number $\ell=1$ are depicted. (a) Three-dimensional plot of $m^{2} \mathcal{V}_{1}$. (b) Contour plot. 'blue' $\rightarrow$ 'red' corresponds to 'high' $\rightarrow$ 'low'.

- Spin zero scalar field: The potential now becomes

$$
\mathcal{V}_{0}=\left(1-\frac{2 m \mathrm{e}^{-a / r}}{r}\right)\left\{\frac{\ell(\ell+1)}{r^{2}}+\frac{2 m \mathrm{e}^{-a / r}}{r^{3}}\left(1-\frac{a}{r}\right)\right\},
$$

and fixing the dominant multipole number $\ell=0$, one specialises to the scalar $s$-wave, which is of particular importance, yielding

$$
\left.\mathcal{V}_{0}\right|_{\ell=0}=\frac{2 m \mathrm{e}^{-a / r}}{r^{3}}\left(1-\frac{2 m \mathrm{e}^{-a / r}}{r}\right)\left(1-\frac{a}{r}\right) \text {. }
$$

Once again, to examine the qualitative features of the potential it is convenient to re-express this in terms of the dimensionless variables $x=r / m, y=a / m$ :

$$
\left.m^{2} \mathcal{V}_{0}\right|_{\ell=0}=\frac{2 \mathrm{e}^{-y / x}}{x^{3}}\left(1-\frac{2 \mathrm{e}^{-y / x}}{x}\right)\left(1-\frac{y}{x}\right) .
$$

The qualitative features of $\mathcal{V}_{0}$ are then displayed in Figure 2.

The most notable feature is the spin zero peak; we see a slight shift in the peak locations between the spin one and spin zero potentials. The green line in Figure $2 b$ is a 'line of best fit', obtained via manual corrections starting from the approximate location of the photon sphere as found in reference [42], and marks the $a$-dependent coordinate location $r_{0} \approx \frac{41}{15} m-\frac{4}{3} a$. Given one does not have information concerning how the peak shifts when comparing the spin one and spin zero potentials a priori, and the peak location is not analytically solvable (see Section 3), this approximation is the best one can do in order to retain the desired level of mathematical tractability. Consequently, in Section 3, the approximation for $r_{0}$ as above is used in the computation of the relevant QNM profiles. The remaining features of the plot are qualitatively similar to those for the spin one case. 

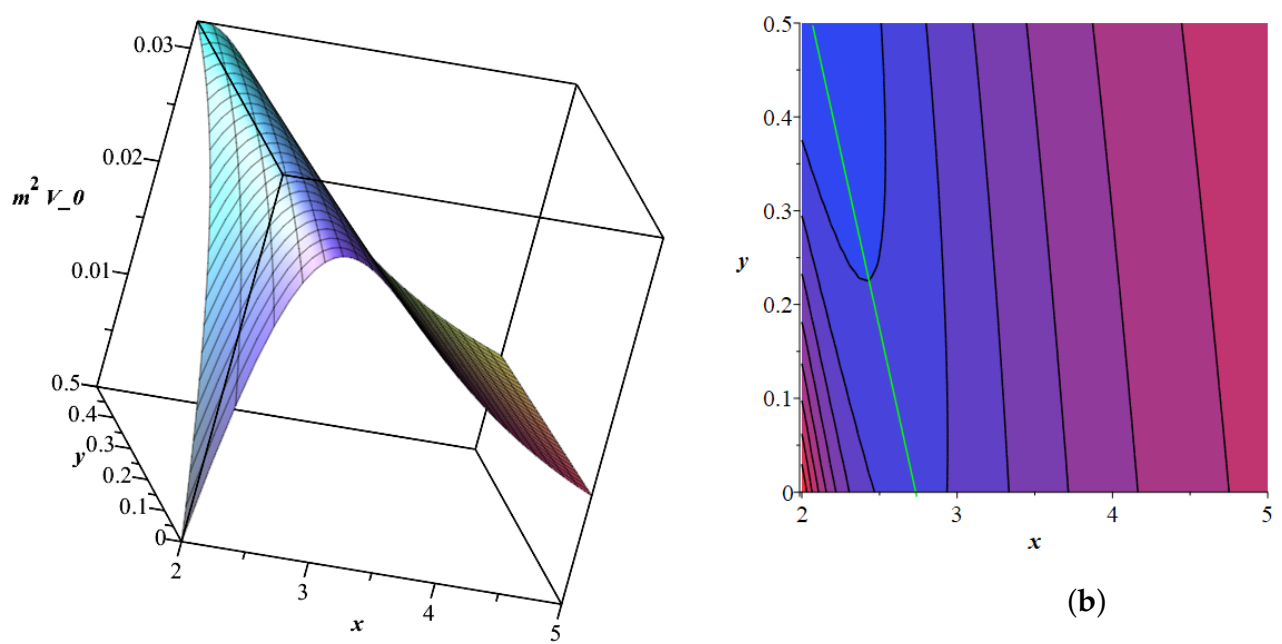

(b)

(a)

Figure 2. The qualitative features of the spin zero Regge-Wheeler potential for the dominant multipole number $\ell=0$ are depicted. (a) Three-dimensional plot of $m^{2} \mathcal{V}_{0}$. (b) Contour plot. 'blue' $\rightarrow$ 'red' corresponds to 'high' $\rightarrow$ 'low'.

- Spin two bivector field (axial mode): The potential becomes

$$
\mathcal{V}_{2}=\left(1-\frac{2 m \mathrm{e}^{-a / r}}{r}\right)\left\{\frac{\ell(\ell+1)}{r^{2}}-\frac{2 m \mathrm{e}^{-a / r}}{r^{3}}\left(3-\frac{a}{r}\right)\right\},
$$

and, fixing the dominant multipole number $\ell=2$, one finds:

$$
\left.\mathcal{V}_{2}\right|_{\ell=2}=\frac{1}{r^{2}}\left(1-\frac{2 m \mathrm{e}^{-a / r}}{r}\right)\left\{6-\frac{2 m \mathrm{e}^{-a / r}}{r}\left(3-\frac{a}{r}\right)\right\} .
$$

Once again, it is informative to re-express this in terms of the dimensionless variables $x=r / m, y=a / m$, giving

$$
\left.m^{2} \mathcal{V}_{2}\right|_{\ell=2}=\frac{1}{x^{2}}\left(1-\frac{2 \mathrm{e}^{-y / x}}{x}\right)\left\{6-\frac{2 \mathrm{e}^{-y / x}}{x}\left(3-\frac{y}{x}\right)\right\}
$$

The qualitative features of $\mathcal{V}_{2}$ are then displayed in Figure 3.

Once again the approximate location for the peak of the spin two (axial) potential is obtained via application of manual corrections to the approximate location of the photon sphere as obtained in reference [42], and is found to be $r_{2} \approx \frac{10}{3} m-\frac{5}{3} a$ (this is the green line in Figure $3 b$ ). This approximation would serve as a starting point to extract QNM profile approximations for the spin two axial mode, similarly to the processes performed for spins one and zero in Section 3. However, for a combination of readability and tractability, this is for now a topic for future research. The remaining qualitative features of the spin two (axial) potential are similar to those for spins one and zero. 


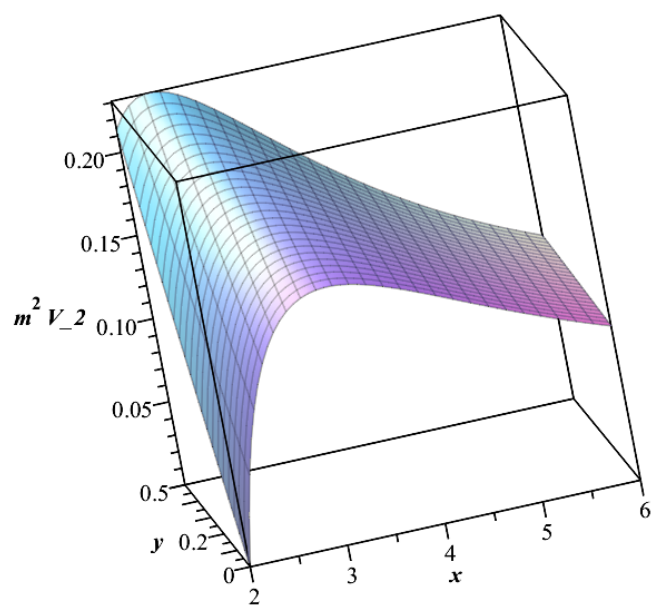

(a)

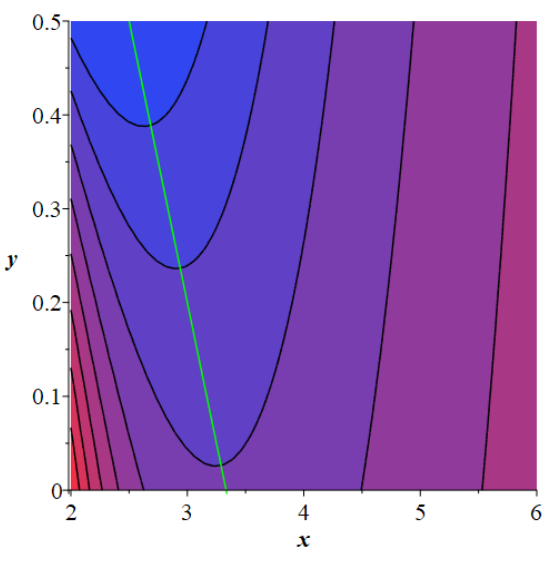

(b)

Figure 3. The qualitative features of the spin two axial Regge-Wheeler potential for the dominant multipole number $\ell=2$ are depicted. (a) Three-dimensional plot of $m^{2} \mathcal{V}_{2}$. (b) Contour plot. 'blue' $\rightarrow$ 'red' corresponds to 'high' $\rightarrow$ 'low'.

\section{First-Order WKB Approximation of the Quasi-Normal Modes}

To calculate the quasi-normal modes for the candidate spacetime, one first defines them in the standard way: they are the $\omega$ present in the right-hand-side of Equation (5), and they satisfy the "radiation" boundary conditions that $\Psi$ is purely outgoing at spatial infinity and purely ingoing at the horizon $[12,23]$. Due to the inherent difficulty of analytically solving the Regge-Wheeler equation, a standard approach in the literature is to use the WKB approximation. Although the WKB method was originally constructed to solve Schrödinger-type equations in quantum mechanics, the close resemblance between the Regge-Wheeler equation Equation (6) and the Schrödinger equation allows for it to be readily adapted to the general relativistic setting. Given the use of the WKB approximation, one cannot extend the analysis of the QNMs for the candidate spacetime to the case when $a>2 \mathrm{~m} / \mathrm{e}$, as for this case there are no horizons in the geometry. The existence of the outer horizon (or at the very least an extremal horizon) is critical to setting up the correct radiative boundary conditions. Other techniques for approximating the QNMs, e.g., time domain integration (see reference [26] for an example), are likely to be applicable in this context. For now, this research is relegated to the domain of the future.

To proceed with the WKB method, one makes the stationary ansatz $\Psi \sim \mathrm{e}^{i \omega t}$, such that all of the qualitative behaviour for $\Psi$ is encoded in the profiles of the respective $\omega$. Computing a WKB approximation to first-order yields a relatively simple and tractable approximation to the quasi-normal modes for a black hole spacetime [12,23,25]:

$$
\omega^{2} \approx\left[\mathcal{V}\left(r_{*}\right)-i\left(n+\frac{1}{2}\right) \sqrt{-2 \partial_{r_{*}}^{2} \mathcal{V}\left(r_{*}\right)}\right]_{r_{*}=r_{\max }},
$$

where $n \in \mathbb{N}$ is the overtone number, and where $r_{*}=r_{\max }$ is the tortoise coordinate location which maximises the relevant Regge-Wheeler potential. It is worth noting that $\left.\mathcal{V}\left(r_{*}\right)\right|_{r_{*}=r_{\max }}=\left.\mathcal{V}(r)\right|_{r_{r=r_{\max }}}$; this will be used in the subsequent analysis.

In-depth calculations of the WKB approximation up to higher orders in a general setting can also be found in references [12,23,25]. Furthermore, various improvements and refinements to the WKB approximation have been explored in references [65-70]. These include the derivation of a generalised higher-order formula, and the exploration of an improved variant of the original WKB approximation using Padé approximants. These formulae present various intractabilities by hand and are best handled by numerical code. 
Consequently, a first-order calculation is performed to holistically analyse the qualitative aspects of the QNMs; refinement in accuracy is left to the numerical relativity community to explore further.

\subsection{Spin One}

For spin one particles recall that the relevant Regge-Wheeler potential is given by

$$
\mathcal{V}_{1}(r)=\left(1-\frac{2 m \mathrm{e}^{-a / r}}{r}\right) \frac{\ell(\ell+1)}{r^{2}} .
$$

The $\mathcal{V}_{1}(r)$ Regge-Wheeler potential is proportional to the $V_{0}(r)$ effective potential used for determining the location of the photon sphere for massless particles [42]. Specifically, one has

$$
\frac{\partial \mathcal{V}_{1}}{\partial r}=\frac{2 \ell(\ell+1)}{r^{3}}\left\{\frac{m \mathrm{e}^{-a / r}}{r}\left(3-\frac{a}{r}\right)-1\right\} .
$$

The resulting stationary points are not analytically solvable, and via comparison with reference [42] one sees that the spin one Regge-Wheeler potential is maximised precisely at the location of the photon sphere; $r_{1}=r_{\gamma}=\sqrt{m \mathrm{e}^{-a / r_{\gamma}}\left(3 r_{\gamma}-a\right)} \approx 3 m-\frac{4}{3} a$. Thus, one immediately obtains the spin one, first-order WKB approximation for the real part of the quasi-normal modes (recall $y=a / m$ ):

$$
\begin{aligned}
\left.\operatorname{Re}\left(\omega^{2}\right) \approx \mathcal{V}_{1}\right|_{r=r_{1}} & \approx \frac{9 \ell(\ell+1)}{(9 m-4 a)^{2}}\left\{1-\frac{6 m \mathrm{e}^{\frac{3 a}{4 a-9 m}}}{9 m-4 a}\right\} \\
& =\frac{9 \ell(\ell+1)}{m^{2}}\left\{\frac{1}{(9-4 y)^{2}}\left[1-\frac{6 \mathrm{e}^{\frac{3 y}{4 y-9}}}{9-4 y}\right]\right\} .
\end{aligned}
$$

Letting $r_{c}=3 m-\frac{4}{3} a$, recalling $x=r / m$ (i.e., $x_{c}=r_{c} / m$ ), and defining $z=a / r_{c}$, alternative representations include (eliminating $m$ ):

$$
\operatorname{Re}\left(\omega^{2}\right) \approx \frac{\ell(\ell+1)}{r_{c}^{2}}\left\{1-\frac{2 \mathrm{e}^{-a / r_{c}}\left(r_{c}+\frac{4}{3} a\right)}{3 r_{c}}\right\}=\frac{\ell(\ell+1)}{r_{c}^{2}}\left\{1-\frac{2 \mathrm{e}^{-z}\left(1+\frac{4}{3} z\right)}{3}\right\},
$$

or (eliminating $a$ ):

$$
\operatorname{Re}\left(\omega^{2}\right) \approx \frac{\ell(\ell+1)}{r_{c}^{2}}\left\{1-\frac{2 m \mathrm{e}^{\frac{3\left(r_{c}-3 m\right)}{4 r_{c}}}}{r_{c}}\right\}=\frac{\ell(\ell+1)}{r_{c}^{2}}\left\{1-\frac{2 \mathrm{e}^{\frac{3}{4}\left(1-\frac{3}{x_{c}}\right)}}{x_{c}}\right\} .
$$

Which expression is preferred is a matter of taste and context.

Now, to compute the imaginary part of the QNMs, note that

$$
\left.\frac{\partial^{2} \mathcal{V}_{1}}{\partial r_{*}^{2}}\right|_{r=r_{1}}=\left.\left(1-\frac{2 m \mathrm{e}^{-a / r}}{r}\right)\left\{\left(1-\frac{2 m \mathrm{e}^{-a / r}}{r}\right) \frac{\partial^{2} \mathcal{V}_{1}}{\partial r^{2}}+\frac{2 m \mathrm{e}^{-a / r}(r-a)}{r^{3}} \frac{\partial \mathcal{V}_{1}}{\partial r}\right\}\right|_{r=r_{1}}
$$

However, already it is known that $\partial \mathcal{V}_{1} /\left.\partial r\right|_{r=r_{1}}=0$, and so this reduces to

$$
\left.\frac{\partial^{2} \mathcal{V}_{1}}{\partial r_{*}^{2}}\right|_{r=r_{1}}=\left.\left(1-\frac{2 m \mathrm{e}^{-a / r}}{r}\right)^{2} \frac{\partial^{2} \mathcal{V}_{1}}{\partial r^{2}}\right|_{r=r_{1}}
$$


Thus, for spin one particles one finds

$$
\begin{gathered}
\left.\frac{\partial^{2} \mathcal{V}_{1}}{\partial r_{*}^{2}}\right|_{r=r_{1}}=\left.\ell(\ell+1)\left(1-\frac{2 m \mathrm{e}^{-a / r}}{r}\right)^{2}\left\{\frac{6}{r^{4}}-(a-2 r)(a-6 r) \frac{2 m \mathrm{e}^{-a / r}}{r^{7}}\right\}\right|_{r=r_{1}} \\
\approx \frac{486 \ell(\ell+1)}{(9 m-4 a)^{4}}\left(1-\frac{6 m \mathrm{e}^{\frac{3 a}{4 a-9 m}}}{9 m-4 a}\right)^{2} \\
\times\left\{1-\frac{27 m \mathrm{e}^{\frac{3 a}{4 a-9 m}}(18 m-11 a)(2 m-a)}{(9 m-4 a)^{3}}\right\},
\end{gathered}
$$

therefore giving

$$
\begin{aligned}
\operatorname{Im}\left(\omega^{2}\right) \approx & -\left.\left(n+\frac{1}{2}\right) \sqrt{-2 \partial_{r_{*}}^{2} \mathcal{V}_{1}(r)}\right|_{r=r_{1}} \\
\approx & -\frac{18 \sqrt{3}}{(9 m-4 a)^{2}}\left(n+\frac{1}{2}\right)\left(1-\frac{6 m \mathrm{e}^{\frac{3 a}{4 a-9 m}}}{9 m-4 a}\right) \\
& \times \sqrt{\ell(\ell+1)\left\{\frac{27 m \mathrm{e}^{\frac{3 a}{4 a-9 m}}(18 m-11 a)(2 m-a)}{(9 m-4 a)^{3}}-1\right\}} \\
=- & \frac{\left(n+\frac{1}{2}\right)}{m^{2}} 18 \sqrt{3}\left(1-\frac{6 \mathrm{e}^{\frac{3 y}{4 y-9}}}{9-4 y}\right) \\
& \times \sqrt{\frac{\ell(\ell+1)}{(9-4 y)^{4}}\left\{\frac{27 \mathrm{e}^{\frac{3 y}{4 y-9}}(18-11 y)(2-y)}{(9-4 y)^{3}}-1\right\}}
\end{aligned}
$$

Alternative expressions include (eliminating $m$ ):

$$
\begin{aligned}
\operatorname{Im}\left(\omega^{2}\right) \approx & -\frac{2 \sqrt{3}\left(n+\frac{1}{2}\right)}{r_{c}^{2}}\left(1-\frac{2 \mathrm{e}^{-a / r_{c}}\left(3 r_{c}+4 a\right)}{9 r_{c}}\right) \\
& \times \sqrt{\ell(\ell+1)\left\{\frac{\left(3 r_{c}+4 a\right)\left(2 r_{c}-a\right)\left(6 r_{c}-a\right) \mathrm{e}^{-a / r_{c}}}{27 r_{c}^{3}}-1\right\}} \\
= & -\frac{2 \sqrt{3}\left(n+\frac{1}{2}\right)}{r_{c}^{2}}\left(1-\frac{2 \mathrm{e}^{-z}(3+4 z)}{9}\right) \\
& \times \sqrt{\ell(\ell+1)\left\{\frac{(3+4 z)(2-z)(6-z) \mathrm{e}^{-z}}{27}-1\right\}},
\end{aligned}
$$


or (eliminating $a)$ :

$$
\begin{aligned}
\operatorname{Im}\left(\omega^{2}\right) \approx-\frac{2 \sqrt{3}\left(n+\frac{1}{2}\right)}{r_{c}^{2}}\left(1-\frac{2 m \mathrm{e}^{\frac{3\left(r_{c}-3 m\right)}{4 r_{c}}}}{r_{c}}\right) \\
\times \sqrt{\ell(\ell+1)\left\{\frac{3 m \mathrm{e}^{\frac{3\left(r_{c}-3 m\right)}{4 r_{c}}}\left(11 r_{c}-9 m\right)\left(3 r_{c}-m\right)}{16 r_{c}^{3}}-1\right\}} \\
=-\frac{2 \sqrt{3}\left(n+\frac{1}{2}\right)}{r_{c}^{2}}\left(1-\frac{2 \mathrm{e}^{\frac{3}{4}\left(1-\frac{3}{x_{c}}\right)}}{x_{c}}\right) \\
\times \sqrt{\ell(\ell+1)\left\{\frac{3 \mathrm{e}^{\frac{3}{4}\left(1-\frac{3}{x_{c}}\right)}\left(11 x_{c}-9\right)\left(3 x_{c}-1\right)}{16 x_{c}^{3}}-1\right\}} .
\end{aligned}
$$

Thus, the first-order WKB approximation of the spin one QNMs, for general multipole numbers $\ell$ and oscillation modes $n$, is given by

$$
\begin{aligned}
\omega^{2} \approx & \frac{9 \ell(\ell+1)}{(9 m-4 a)^{2}}\left(1-\frac{6 m \mathrm{e}^{\frac{3 a}{4 a-9 m}}}{9 m-4 a}\right) \\
& \times\left\{1-2 \sqrt{3} i\left(n+\frac{1}{2}\right) \sqrt{\frac{1}{\ell(\ell+1)}\left[\frac{27 m \mathrm{e}^{\frac{3 a}{4 a-9 m}}(18 m-11 a)(2 m-a)}{(9 m-4 a)^{3}}-1\right]}\right. \\
= & \frac{9 \ell(\ell+1)}{m^{2}(9-4 y)^{2}}\left(1-\frac{6 \mathrm{e}^{\frac{3 y}{4 y-9}}}{9-4 y}\right) \\
& \times\left\{1-2 \sqrt{3} i\left(n+\frac{1}{2}\right) \sqrt{\left.\frac{1}{\ell(\ell+1)}\left[\frac{27 \mathrm{e}^{\frac{3 y}{4 y-9}}(18-11 y)(2-y)}{(9-4 y)^{3}}-1\right]\right\} .}\right.
\end{aligned}
$$

For the purposes of extracting numerical results in Section 4, it is useful to redefine this as the dimensionless quantity

$$
\begin{aligned}
m^{2} \omega^{2} \approx & \frac{9 \ell(\ell+1)}{(9-4 y)^{2}}\left(1-\frac{6 \mathrm{e}^{\frac{3 y}{4 y-9}}}{9-4 y}\right) \\
& \times\left\{1-2 \sqrt{3} i\left(n+\frac{1}{2}\right) \sqrt{\frac{1}{\ell(\ell+1)}\left[\frac{27 \mathrm{e}^{\frac{3 y}{4 y-9}}(18-11 y)(2-y)}{(9-4 y)^{3}}-1\right]} .\right.
\end{aligned}
$$


Alternative expressions include (eliminating $m$ ):

$$
\begin{aligned}
\omega^{2} \approx & \frac{\ell(\ell+1)}{r_{c}^{2}}\left(1-\frac{2\left(3 r_{c}+4 a\right) \mathrm{e}^{-a / r_{c}}}{9 r_{c}}\right) \\
& \times\left\{1-2 \sqrt{3} i\left(n+\frac{1}{2}\right) \sqrt{\frac{1}{\ell(\ell+1)}\left[\frac{\left(2 r_{c}-a\right)\left(6 r_{c}-a\right)\left(3 r_{c}+4 a\right) \mathrm{e}^{-a / r_{c}}}{27 r_{c}^{3}}-1\right]}\right. \\
= & \frac{\ell(\ell+1)}{r_{c}^{2}}\left(1-\frac{2(3+4 z) \mathrm{e}^{-z}}{9}\right) \\
& \times\left\{1-2 \sqrt{3} i\left(n+\frac{1}{2}\right) \sqrt{\frac{1}{\ell(\ell+1)}\left[\frac{(3+4 z)(2-z)(6-z) \mathrm{e}^{-z}}{27}-1\right]},\right.
\end{aligned}
$$

or (eliminating $a$ ):

$$
\begin{aligned}
\omega^{2} \approx & \frac{\ell(\ell+1)}{r_{c}^{2}}\left(1-\frac{2 m \mathrm{e}^{\frac{3\left(r_{c}-3 m\right)}{4 r_{c}}}}{r_{c}}\right) \\
& \times\left\{1-2 \sqrt{3} i\left(n+\frac{1}{2}\right) \sqrt{\left.\frac{1}{\ell(\ell+1)}\left[\frac{3 m \mathrm{e}^{\frac{3\left(r_{c}-3 m\right)}{4 r_{c}}}\left(11 r_{c}-9 m\right)\left(3 r_{c}-m\right)}{16 r_{c}^{3}}-1\right]\right\}}\right. \\
= & \frac{\ell(\ell+1)}{r_{c}^{2}}\left(1-\frac{2 \mathrm{e}^{\frac{3}{4}\left(1-\frac{3}{x_{c}}\right)}}{x_{c}}\right) \\
& \times\left\{1-2 \sqrt{3} i\left(n+\frac{1}{2}\right) \sqrt{\frac{1}{\ell(\ell+1)}\left[\frac{3 \mathrm{e}^{\frac{3}{4}\left(1-\frac{3}{x_{c}}\right)}\left(11 x_{c}-9\right)\left(3 x_{c}-1\right)}{16 x_{c}^{3}}-1\right]} .\right.
\end{aligned}
$$

In the Schwarzschild limit one obtains

$$
\omega_{\text {Sch. }}^{2}=\lim _{a \rightarrow 0}\left(\omega^{2}\right) \approx \frac{\ell(\ell+1)}{27 m^{2}}\left(1-\frac{i(2 n+1)}{\sqrt{\ell(\ell+1)}}\right),
$$

which agrees with existing work in the literature [12,23].

\subsection{Spin Zero}

For spin zero particles recall that one has the following specific form for the ReggeWheeler potential:

$$
\mathcal{V}_{0}(r)=\left(1-\frac{2 m \mathrm{e}^{-a / r}}{r}\right)\left\{\frac{\ell(\ell+1)}{r^{2}}+\frac{2 m \mathrm{e}^{-a / r}\left(1-\frac{a}{r}\right)}{r^{3}}\right\} .
$$

It is immediately clear that the peak of this potential is going to be slightly shifted from the location of the photon sphere, which for the spin one case maximised $\mathcal{V}_{1}(r)$. Computing:

$$
\begin{aligned}
\frac{\partial \mathcal{V}_{0}}{\partial r}= & \frac{1}{r^{3}}\left\{\left[\frac{2 m \mathrm{e}^{-a / r}}{r}\left(3-\frac{a}{r}\right)-2\right]\left[\ell(\ell+1)+\frac{2 m \mathrm{e}^{-a / r}}{r}\left(1-\frac{a}{r}\right)\right]\right. \\
& \left.+\left(1-\frac{2 m \mathrm{e}^{-a / r}}{r}\right)\left[\frac{-2 m \mathrm{e}^{-a / r}}{r}\left(\left(\frac{a}{r}\right)^{2}-3\left(\frac{a}{r}\right)+1\right)\right]\right\} .
\end{aligned}
$$


The associated stationary points are not analytically solvable for $r$. It is worth noting that in general, the stationary points of $\mathcal{V}_{0}$ are $\ell$-dependent, unlike in the case for $\mathcal{V}_{1}$. Without knowledge of the location of the peak for the spin zero potential a priori, the best line of inquiry which retains the desired level of tractability is to specialise to the scalar $s$-wave (corresponding to $\ell=0$ ), which is of particular importance, playing a dominant role in the signal. This constraint is fit for purpose in extracting the relevant results in Section 4. Specialising to the s-wave, one finds

$$
\left.\frac{\partial \mathcal{V}_{0}}{\partial r}\right|_{\ell=0}=\frac{2 m \mathrm{e}^{-a / r}}{r^{4}}\left\{\frac{m \mathrm{e}^{-a / r}}{r}\left[4\left(\frac{a}{r}\right)^{2}-14\left(\frac{a}{r}\right)+8\right]-\left[\left(\frac{a}{r}\right)^{2}-5\left(\frac{a}{r}\right)+3\right]\right\},
$$

and still the associated stationary points are not analytically solvable. As such, to make progress one uses the approximate location of the peak as found in Section $2 ; r_{0} \approx \frac{41}{15} m-\frac{4}{3} a$. See Figure $2 b$ for details. One obtains the following approximation for the real part of the spin zero QNMs for the scalar s-wave:

$$
\begin{aligned}
\left.\operatorname{Re}\left(\omega^{2}\right) \approx \mathcal{V}_{0}\left(r_{0}\right)\right|_{\ell=0} & \approx \frac{6750 m \mathrm{e}^{\frac{15 a}{20 a-41 m}}(41 m-35 a)\left(30 m \mathrm{e}^{\frac{15 a}{20 a-41 m}}+20 a-41 m\right)}{(20 a-41 m)^{5}} \\
& =\frac{6750}{m^{2}}\left[\frac{\mathrm{e}^{\frac{15 y}{20 y-41}}(41-35 y)\left(30 \mathrm{e}^{\frac{15 y}{20 y-41}}+20 y-41\right)}{(20 y-41)^{5}}\right] .
\end{aligned}
$$

For the imaginary part of the spin zero QNMs, first one has

$$
\left.\frac{\partial^{2} \mathcal{V}_{0}}{\partial r_{*}^{2}}\right|_{r=r_{0}}=\left.\left(1-\frac{2 m \mathrm{e}^{-a / r}}{r}\right)^{2} \frac{\partial^{2} \mathcal{V}_{0}}{\partial r^{2}}\right|_{r=r_{0}}
$$

with

$$
\begin{aligned}
& \frac{\partial^{2} \mathcal{V}_{0}}{\partial r^{2}}=\frac{2}{r^{4}}\left\{3 \ell(\ell+1)+\frac{4 m^{2} \mathrm{e}^{-2 a / r}}{r^{2}}\left[2\left(\frac{a}{r}\right)^{2}-10\left(\frac{a}{r}\right)+5\right]\left(\frac{a}{r}-2\right)\right. \\
& -\frac{m \mathrm{e}^{-a / r}}{r}\left[12\left(\ell^{2}+\ell-1\right)-4\left(\frac{a}{r}\right)\left(2 \ell^{2}+2 \ell-7\right)\right. \\
& \left.\left.+\left(\frac{a}{r}\right)^{2}\left(\ell^{2}+\ell-11\right)+\left(\frac{a}{r}\right)^{3}\right]\right\}
\end{aligned}
$$

giving

$$
\begin{array}{r}
\frac{\partial^{2} \mathcal{V}_{0}}{\partial r_{*}^{2}}=\frac{2}{r^{4}}\left(1-\frac{2 m \mathrm{e}^{-a / r}}{r}\right)^{2}\left\{3 \ell(\ell+1)+\frac{4 m^{2} \mathrm{e}^{-2 a / r}}{r^{2}}\left[2\left(\frac{a}{r}\right)^{2}-10\left(\frac{a}{r}\right)+5\right]\left(\frac{a}{r}-2\right)\right. \\
-\frac{m \mathrm{e}^{-a / r}}{r}\left[12\left(\ell^{2}+\ell-1\right)-4\left(\frac{a}{r}\right)\left(2 \ell^{2}+2 \ell-7\right)\right. \\
\left.\left.+\left(\frac{a}{r}\right)^{2}\left(\ell^{2}+\ell-11\right)+\left(\frac{a}{r}\right)^{3}\right]\right\} .
\end{array}
$$


For tractability, it is now prudent to specialise to $\ell=0$. This yields

$$
\begin{aligned}
\left.\frac{\partial^{2} \mathcal{V}_{0}}{\partial r_{*}^{2}}\right|_{\ell=0}= & \frac{2}{r^{4}}\left(1-\frac{2 m \mathrm{e}^{-a / r}}{r}\right)^{2}\left\{\left(\frac{2 m \mathrm{e}^{-a / r}}{r}\right)^{2}\left(\frac{a}{r}-2\right)\left[2\left(\frac{a}{r}\right)^{2}-10\left(\frac{a}{r}\right)+5\right]\right. \\
& \left.-\frac{m \mathrm{e}^{-a / r}}{r}\left[-12+28\left(\frac{a}{r}\right)-11\left(\frac{a}{r}\right)^{2}+\left(\frac{a}{r}\right)^{3}\right]\right\},
\end{aligned}
$$

and one obtains the following approximation for the imaginary part of the $s$-wave spin zero QNMs (expressed only as a function of $y$ for readability):

$$
\begin{aligned}
\operatorname{Im}\left(\omega^{2}\right) \approx & -\left.\left(n+\frac{1}{2}\right) \sqrt{-2\left[\partial_{r_{*}}^{2} \mathcal{V}_{0}\left(r_{0} \approx \frac{41}{15} m-\frac{4}{3} a\right)\right]}\right|_{\ell=0} \\
= & -\frac{1350}{m^{2}}\left(n+\frac{1}{2}\right)\left[\frac{\sqrt{5} \mathrm{e}^{\frac{15 y}{20 y-41}}\left(30 \mathrm{e}^{\frac{15 y}{20 y-41}}+20 y-41\right)}{(20 y-41)^{\frac{11}{2}}}\right] \\
& \times\left\{100 \mathrm{e}^{\frac{15 y}{20 y-41}}\left(59950 y^{3}-247230 y^{2}+327795 y-137842\right)\right. \\
+ & \left.2112500 y^{4}-13535125 y^{3}+31644825 y^{2}-31703660 y+11303044\right\}^{\frac{1}{2}} .
\end{aligned}
$$

Combining Equations (43) and (48) gives the approximation for the dimensionful $\omega^{2}$; however the expression is unwieldy and not particularly important to display here. Using Equations (43) and (48) to compute the real and imaginary approximations for the dimensionless $m^{2} \omega^{2}$, respectively, is sufficient for extracting the relevant results in Section 4.

\section{Numerical Results}

Given that the behaviour of the waveform is aggressively governed by the fundamental mode, to extract profile approximations it is both physically well-motivated and mathematically tractable to specialise to $n=0$. Given the WKB approximation is only valid in the presence of a nontrivial horizon structure, and that the candidate spacetime only possesses horizons for $a \in\left(0, \frac{2 m}{\mathrm{e}}\right)$, it is prudent for one to define the dimensionless object $\hat{a}=a / a_{\max }=\frac{a \mathrm{e}}{2 m}=\frac{\mathrm{e}}{2} y$, such that $\hat{a} \in(0,1)$. One may then define the dimensionless $\hat{\omega}=\omega m$, such that all of the qualitative information for the dimensionful $\omega$ as a function of the dimensionful $a$ is now encoded in the dimensionless $\hat{\omega}$ as a function of the dimensionless $\hat{a}$. As such, one then examines $\hat{\omega}$ by plugging in values of $y=\frac{2}{\mathrm{e}} \hat{a}$ into the relevant equations from Section 3 on a case-by-case basis. Lastly, it will be of most use to analyse the dominant multipole number in each spin case. Given $\ell \geq S$, for electromagnetic spin one fluctuations this will correspond to analysing $\ell=1$, for scalar spin zero fluctuations one analyses the $s$-wave corresponding to fixing $\ell=0$ (as already stipulated), and finally for spin two axial perturbations one would fix $\ell=2$. Notably this implies that the approximate locations for the peaks of the relevant RW-potentials computed in Section 2 are directly applicable here. 


\subsection{Spin One}

Consequently, to analyse QNM profile approximations for electromagnetic spin one fluctuations on the background spacetime, fix the fundamental mode $n=0$, and analyse the special case of the dominant multipole number $\ell=1$. Substituting these values into Equation (36) and computing the resulting square root gives the results from Table 1 for the approximation of $\hat{\omega}$ for different values of $\hat{a} \in(0,1)$ (rounded to 6 d.p.):

Table 1. Fundamental QNM of the spin one field for $\ell=1$, obtained via first-order WKB approximation.

\begin{tabular}{cc}
\hline$\hat{a}$ & WKB Approx. for $\hat{\boldsymbol{w}}$ \\
\hline 0.0 & $0.287050-0.091235 i$ \\
\hline 0.1 & $0.293902-0.092012 i$ \\
\hline 0.2 & $0.301291-0.092532 i$ \\
\hline 0.3 & $0.309304-0.092708 i$ \\
\hline 0.4 & $0.318051-0.092419 i$ \\
\hline 0.5 & $0.327658-0.091486 i$ \\
\hline 0.6 & $0.338285-0.089636 i$ \\
\hline 0.7 & $0.350117-0.086433 i$ \\
\hline 0.8 & $0.363377-0.081139 i$ \\
\hline 0.9 & $0.378330-0.072338 i$ \\
\hline 1.0 & $0.395289-0.056624 i$ \\
\hline
\end{tabular}

Immediately there are the following qualitative observations:

- As a sanity check, $\operatorname{Im}(\hat{\omega})<0$ for all values of $\hat{a}$, indicating that the propagation of electromagnetic fields in the background spacetime is stable-an expected result;

- $\operatorname{Re}(\hat{\omega})$ increases monotonically with $\hat{a}$-this is the frequency of the corresponding QNMs;

- $\operatorname{Im}(\hat{\omega})$ decreases with $\hat{a}$ initially, up until $\hat{a} \approx 0.35$, and then it increases monotonically with $\hat{a}$ for the remainder of the domain - this is the decay rate or damping rate of the QNMs;

- Given that throughout the historical literature, it is conventional to assert $\hat{a} \sim m_{p}$, it is likely of primary interest to examine the behaviour of this plot for small $\hat{a}$. In view of this, if one constrains the analysis of the qualitative behaviour for $\hat{\omega}$ prior to the trough present in Figure 4, one would expect that the signals for electromagnetic radiation propagating in the presence of a regular black hole with asymptotically Minkowski core should have both a higher frequency as well as a faster decay rate than their Schwarzschild counterparts. This qualitative result may translate to the spin two case, and speak directly to the LIGO/VIRGO calculation. The fact that the signal is expected to be shorter-lived could present a heightened level of experimental difficulty when trying to delineate signals, though this may very well be offset by the fact that the signal also carries higher energy; further discussion on these points is left to both the numerical relativity and experimental communities. 


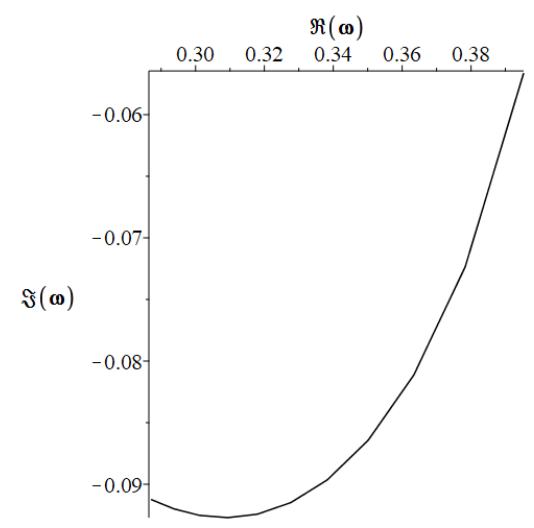

Figure 4. A plot of the points from Table 1 with a linear interpolation curve. $\operatorname{Re}(\hat{\omega})$ increases monotonically with $\hat{a}$, hence increasing $\hat{a}$ corresponds to one moving from left to right.

\subsection{Spin Zero}

For spin zero scalar fluctuations, specialising to the s-wave, similarly fix the fundamental mode $n=0$. Substituting this into Equations (43) and (48), which are the relevant equations to compute the real and imaginary approximations of $\hat{\omega}^{2}$, respectively, (recall these have already specialised to the $s$-wave given $\ell$ has already been fixed to be zero), and then taking the appropriate square root yields the results from Table 2 and Figure 5 (to 6 d.p.):

Table 2. Fundamental QNM of the massless, minimally coupled spin zero scalar field for the s-wave $(\ell=0)$, obtained via first-order WKB approximation.

\begin{tabular}{cc}
\hline$\hat{\boldsymbol{a}}$ & WKB Approx. for $\hat{\boldsymbol{w}}$ \\
\hline 0.0 & $0.187409-0.094054 i$ \\
\hline 0.1 & $0.189734-0.094530 i$ \\
\hline 0.2 & $0.191948-0.094669 i$ \\
\hline 0.3 & $0.194049-0.094425 i$ \\
\hline 0.4 & $0.196027-0.093742 i$ \\
\hline 0.5 & $0.197868-0.092557 i$ \\
\hline 0.6 & $0.199552-0.090796 i$ \\
\hline 0.7 & $0.201042-0.088385 i$ \\
\hline 0.8 & $0.202285-0.085306 i$ \\
\hline 0.9 & $0.203235-0.081735 i$ \\
\hline 1.0 & $0.203894-0.078421 i$ \\
\hline
\end{tabular}




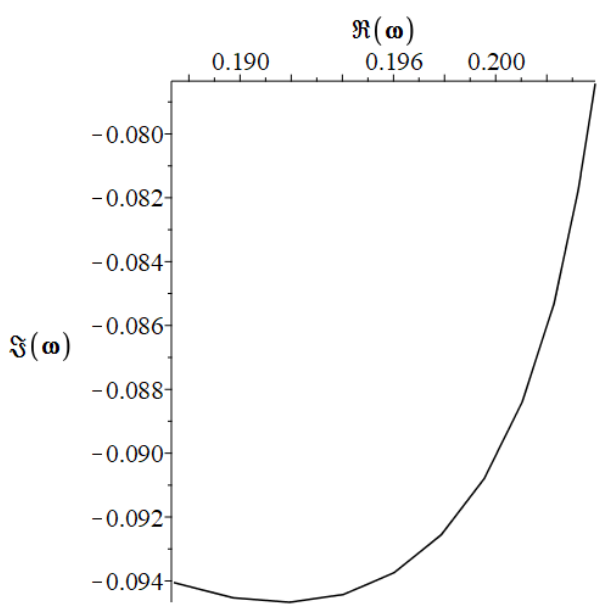

Figure 5. A plot of the points from Table 2 with a linear interpolation curve. $\operatorname{Re}(\hat{\omega})$ increases monotonically with $\hat{a}$, hence increasing $\hat{a}$ corresponds to one moving from left to right.

There are the following qualitative observations:

- $\operatorname{Re}(\hat{\omega})$ once again increases monotonically with $\hat{a}-$ higher $\hat{a}$-values correspond to higher frequency fundamental modes;

- $\operatorname{Im}(\hat{\omega})<0$ for all $\hat{a}$, indicating that the $s$-wave for minimally coupled massless scalar fields propagating in the background spacetime is stable;

- $\operatorname{Im}(\hat{\omega})$ decreases with $\hat{a}$ initially (down to a trough around $\hat{a}=0.25$ ), before monotonically increasing with $\hat{a}$ for the rest of the domain — this is the decay/damping rate of the QNMs;

- Similarly as for the electromagnetic spin one case, when one examines the behaviour for small $\hat{a}$, the signals for the fundamental mode of spin zero scalar field perturbations in the presence of a regular black hole with asymptotically Minkowski core are expected to have a higher frequency and to be shorter-lived than for their Schwarzschild counterparts.

\subsection{Comparison with Bardeen and Hayward}

It is worth investigating whether these qualitative results are aligned with the analogous results for other well-known regular black hole geometries in GR. Analysis of the QNMs for both the Bardeen [33] and Hayward [34] regular black holes has been performed in references [18-21]. The choices made in setting up a tractable numerical analysis make it difficult to directly compare many of the findings; however in Appendix A of reference [18], some analogous and comparable results are presented for the spin zero case for both the Bardeen and Hayward models. The findings can be summarised as follows:

- For the fundamental mode of the spin zero scalar s-wave for the Bardeen regular black hole, as deviation from Schwarzschild increases, $\operatorname{Re}(\omega)$ increases and $|\operatorname{Im}(\omega)|$ decreases. The signals are hence expected to have higher frequency but be longer-lived than for their Schwarzschild counterparts;

- For the fundamental mode of the spin zero scalar $s$-wave for the Hayward regular black hole, as deviation from Schwarzschild increases, both $\operatorname{Re}(\omega)$ and $|\operatorname{Im}(\omega)|$ decrease. The signals are hence expected to have lower frequency and be longer-lived than for their Schwarzschild counterparts.

These results suggest that for spin zero perturbations, one does not have the same qualitative differences in the ringdown signal between the class of regular black hole models in static spherical symmetry and Schwarzschild. Consequently, the ability to delineate between singular and nonsingular astrophysical sources based on observed signals by LIGO/VIRGO (or LISA) is likely a question of comparing specific candidate geometries, rather than comparing the bracket of 'regular spacetimes' to their singular counterparts. Whether this extends to the more astrophysically relevant domain of axisymmetry, or in- 
deed to spin two axial and polar perturbations, is at this stage unclear. Furthermore, given that the parameters which quantify the deviation from Schwarzschild are often associated with quantum scales, one conjectures that the current margin of error present in the data from LIGO/VIRGO is too high to be able to form robust conclusions; this is left to the numerical and experimental community for further comment. LISA is far more likely to be able to probe with the necessary level of accuracy.

\section{Perturbing the Potential-General First-Order Analysis}

Suppose one perturbs the Regge-Wheeler potential itself, replacing $\mathcal{V}(r) \rightarrow \mathcal{V}(r)+$ $\delta \mathcal{V}(r)$. It is of interest to analyse what effect this has on the estimate for the QNMs. Classical perturbation of the potential to first-order in $\epsilon$ is performed, capturing any linear contributions from external agents that may disturb the propagating waveforms. First-order perturbation is well-motivated from the perspective of the historical literature, and ensures the analysis has the desired level of tractability. As such, one has the following: $\mathcal{V}(r) \rightarrow \mathcal{V}(r)+\delta \mathcal{V}(r)=\mathcal{V}(r)+\epsilon \delta \mathcal{V}_{a}(r)+\epsilon^{2} \delta \mathcal{V}_{b}(r)+\mathcal{O}\left(\epsilon^{3}\right) \approx \mathcal{V}(r)+\epsilon \delta \mathcal{V}_{a}(r)$. All terms of order $\epsilon^{2}$ or higher are therefore truncated. Consequently, for notational convenience it is advantageous to simply replace $\delta \mathcal{V}_{\alpha}(r)$ with $\delta \mathcal{V}(r)$ in the discourse that follows, eliminating superfluous indices. Furthermore, for notational convenience, define $r_{\max }=r_{\sigma}$ to be the generalised location of the peak of the potentials. One observes the following effects on the QNMs:

- First, the position of the peak shifts:

$$
\left.0 \approx[\mathcal{V}+\epsilon \delta \mathcal{V}]^{\prime}(r)\right|_{r=r_{\sigma}+\epsilon \delta r_{\sigma}}
$$

giving

$$
\mathcal{V}^{\prime}\left(r_{\sigma}+\epsilon \delta r_{\sigma}\right)+\epsilon[\delta \mathcal{V}]^{\prime}\left(r_{\sigma}+\epsilon \delta r_{\sigma}\right) \approx 0 .
$$

Performing a first-order Taylor series expansion of the left-hand-side of Equation (50) about $\delta r_{0}=0$ then yields

$$
\mathcal{V}^{\prime}\left(r_{\sigma}\right)+\epsilon[\delta \mathcal{V}]^{\prime}\left(r_{\sigma}\right)+\epsilon \delta r_{\sigma}\left\{\mathcal{V}^{\prime \prime}\left(r_{\sigma}\right)+\epsilon[\delta \mathcal{V}]^{\prime \prime}\left(r_{\sigma}\right)\right\} \approx 0,
$$

and eliminating the term of order $\epsilon^{2}$, combined with the knowledge that $\mathcal{V}^{\prime}\left(r_{\sigma}\right)=0$, gives

$$
\delta r_{\sigma} \approx-\frac{[\delta \mathcal{V}]^{\prime}\left(r_{\sigma}\right)}{\mathcal{V}^{\prime \prime}\left(r_{\sigma}\right)}
$$

- Secondly, the height of the peak shifts:

$$
[\mathcal{V}+\epsilon \delta \mathcal{V}]\left(r_{\sigma}+\epsilon \delta r_{\sigma}\right)=\mathcal{V}\left(r_{\sigma}+\epsilon \delta r_{\sigma}\right)+\epsilon[\delta \mathcal{V}]\left(r_{\sigma}+\epsilon \delta r_{\sigma}\right)
$$

and performing a first-order Taylor series expansion about $\delta r_{0}=0$ yields the following to first-order in $\epsilon$ :

$$
\begin{aligned}
{[\mathcal{V}+\epsilon \delta \mathcal{V}]\left(r_{\sigma}+\epsilon \delta r_{\sigma}\right) } & \approx \mathcal{V}\left(r_{\sigma}\right)+\epsilon\left\{[\delta \mathcal{V}]\left(r_{\sigma}\right)+\delta r_{\sigma} \mathcal{V}^{\prime}\left(r_{\sigma}\right)\right\} \\
& =\mathcal{V}\left(r_{\sigma}\right)+\epsilon[\delta \mathcal{V}]\left(r_{\sigma}\right)
\end{aligned}
$$

- Third, the curvature at the peak shifts

$$
[\mathcal{V}+\epsilon \delta \mathcal{V}]^{\prime \prime}\left(r_{\sigma}+\epsilon \delta r_{\sigma}\right)=\mathcal{V}^{\prime \prime}\left(r_{\sigma}+\epsilon \delta r_{\sigma}\right)+\epsilon[\delta \mathcal{V}]^{\prime \prime}\left(r_{\sigma}+\epsilon \delta r_{\sigma}\right),
$$

which for first-order-Taylor about $\delta r_{\sigma}=0$ and to first-order in $\epsilon$ gives

$$
[\mathcal{V}+\epsilon \delta \mathcal{V}]^{\prime \prime}\left(r_{\sigma}+\epsilon \delta r_{\sigma}\right) \approx \mathcal{V}^{\prime \prime}\left(r_{\sigma}\right)+\epsilon[\delta \mathcal{V}]^{\prime \prime}\left(r_{\sigma}\right)+\epsilon \delta r_{\sigma} \mathcal{V}^{\prime \prime \prime}\left(r_{\sigma}\right)
$$


which from Equation (52) can then be approximated by the following

$$
[\mathcal{V}+\epsilon \delta \mathcal{V}]^{\prime \prime}\left(r_{\sigma}+\epsilon \delta r_{\sigma}\right) \approx \mathcal{V}^{\prime \prime}\left(r_{\sigma}\right)+\epsilon\left\{[\delta \mathcal{V}]^{\prime \prime}\left(r_{\sigma}\right)-\frac{\mathcal{V}^{\prime \prime \prime}\left(r_{\sigma}\right)}{\mathcal{V}^{\prime \prime}\left(r_{\sigma}\right)}[\delta \mathcal{V}]^{\prime}\left(r_{\sigma}\right)\right\} .
$$

Given the following (one assumes the existence of some tortoise coordinate relation $\left.\partial_{r_{*}}=T(r) \partial_{r}\right)$ :

$$
\begin{aligned}
\partial_{r_{*}}^{2}[\mathcal{V}+\epsilon \delta \mathcal{V}]\left(r_{\sigma}+\epsilon \delta r_{\sigma}\right) & =\left.\partial_{r_{*}}\left\{T(r) \partial_{r}[\mathcal{V}+\epsilon \delta \mathcal{V}](r)\right\}\right|_{r=r_{\sigma}+\epsilon \delta r_{\sigma}} \\
& =T^{2}(r)[\mathcal{V}+\epsilon \delta \mathcal{V}]^{\prime \prime}(r)+\left.[\mathcal{V}+\epsilon \delta \mathcal{V}]^{\prime}(r) T^{\prime}(r) T(r)\right|_{r=r_{\sigma}+\epsilon \delta r_{\sigma}},
\end{aligned}
$$

one may perform Taylor series expansions about $\epsilon=0$ for the relevant terms (and truncate to first-order in $\epsilon$ ):

$$
\begin{aligned}
& \partial_{r_{*}}^{2}[\mathcal{V}+\epsilon \delta \mathcal{V}]\left(r_{\sigma}+\epsilon \delta r_{\sigma}\right)= {\left[T\left(r_{\sigma}+\epsilon \delta r_{\sigma}\right)\right]^{2}[\mathcal{V}+\epsilon \delta \mathcal{V}]^{\prime \prime}\left(r_{\sigma}+\epsilon \delta r_{\sigma}\right) } \\
&+[\mathcal{V}+\epsilon \delta \mathcal{V}]^{\prime}\left(r_{\sigma}+\epsilon \delta r_{\sigma}\right) T^{\prime}\left(r_{\sigma}+\epsilon \delta r_{\sigma}\right) T\left(r_{\sigma}+\epsilon \delta r_{\sigma}\right) \\
& \approx\left[T\left(r_{\sigma}\right)+\epsilon \delta r_{\sigma} T^{\prime}\left(r_{\sigma}\right)\right]^{2}[\mathcal{V}+\epsilon \delta \mathcal{V}]^{\prime \prime}\left(r_{\sigma}+\epsilon \delta r_{\sigma}\right) \\
&+\epsilon \delta r_{\sigma} \mathcal{V}^{\prime \prime}\left(r_{\sigma}\right) T^{\prime}\left(r_{\sigma}\right) T\left(r_{\sigma}\right) \\
& \approx\left[T^{2}\left(r_{\sigma}\right)+2 \epsilon \delta r_{\sigma} T^{\prime}\left(r_{\sigma}\right)\right][\mathcal{V}+\epsilon \delta \mathcal{V}]^{\prime \prime}\left(r_{\sigma}+\epsilon \delta r_{\sigma}\right) \\
&-\epsilon T^{\prime}\left(r_{\sigma}\right) T\left(r_{\sigma}\right)[\delta \mathcal{V}]^{\prime}\left(r_{\sigma}\right),
\end{aligned}
$$

and substituting the result from Equation (57) then finally gives (all functions on the right-hand-side are evaluated at $r=r_{\sigma}$; notation suppressed for tractability):

$$
\partial_{r_{*}}^{2}[\mathcal{V}+\epsilon \delta \mathcal{V}] \approx T^{2} \mathcal{V}^{\prime \prime}-\epsilon\left\{\left(T^{\prime} T+2 T^{\prime}+T^{2} \frac{\mathcal{V}^{\prime \prime \prime}}{\mathcal{V}^{\prime \prime}}\right)[\delta \mathcal{V}]^{\prime}-T^{2}[\delta \mathcal{V}]^{\prime \prime}\right\}
$$

As such, for the square root one has:

$$
\begin{aligned}
\sqrt{-2 \partial_{r_{*}}^{2}[\mathcal{V}+\epsilon \delta \mathcal{V}]\left(r_{\sigma}+\epsilon \delta r_{\sigma}\right)} \approx & \left\{-2 T^{2} \mathcal{V}^{\prime \prime}\right. \\
& \left.+2 \epsilon\left[\left(T^{\prime} T+2 T^{\prime}+T^{2} \frac{\mathcal{V}^{\prime \prime \prime}}{\mathcal{V}^{\prime \prime}}\right)[\delta \mathcal{V}]^{\prime}-T^{2}[\delta \mathcal{V}]^{\prime \prime}\right]\right\}^{\frac{1}{2}},
\end{aligned}
$$

and performing a first-order Taylor series expansion about $\epsilon=0$ gives (all functions on the right-hand-side are evaluated at $r=r_{\sigma}$ ):

$$
\begin{aligned}
& \sqrt{-2 \partial_{r_{*}}^{2}[\mathcal{V}+\epsilon \delta \mathcal{V}]} \approx T \sqrt{-2 \mathcal{V}^{\prime \prime}}-\frac{\epsilon}{\sqrt{2}}\left\{\left(\frac{T^{\prime}}{\left|\mathcal{V}^{\prime \prime}\right|^{\frac{1}{2}}}+\frac{2 T^{\prime}}{T\left|\mathcal{V}^{\prime \prime}\right|^{\frac{1}{2}}}+\frac{T \mathcal{V}^{\prime \prime \prime}}{\left|\mathcal{V}^{\prime \prime}\right|^{\frac{3}{2}}}\right)[\delta \mathcal{V}]^{\prime}\right. \\
& \left.-\frac{T}{\left|\mathcal{V}^{\prime \prime}\right|^{\frac{1}{2}}}[\delta \mathcal{V}]^{\prime \prime}\right\}
\end{aligned}
$$


Assembling the pieces, to first-order in WKB and first-order in $\epsilon$ the approximate shift in the QNMs is given by:

$$
\begin{aligned}
\delta\left(\omega_{n}^{2}\right) \approx \epsilon[\delta \mathcal{V}] & \\
& +i\left(n+\frac{1}{2}\right) \frac{\epsilon}{\sqrt{2}}\left\{\left(\frac{T^{\prime}}{\left|\mathcal{V}^{\prime \prime}\right|^{\frac{1}{2}}}+\frac{2 T^{\prime}}{T\left|\mathcal{V}^{\prime \prime}\right|^{\frac{1}{2}}}+\frac{T \mathcal{V}^{\prime \prime \prime}}{\left|\mathcal{V}^{\prime \prime}\right|^{\frac{3}{2}}}\right)[\delta \mathcal{V}]^{\prime}-\frac{T}{\left|\mathcal{V}^{\prime \prime}\right|^{\frac{1}{2}}}[\delta \mathcal{V}]^{\prime \prime}\right\},
\end{aligned}
$$

where all expressions on the right-hand-side are evaluated at $r=r_{\sigma}$. This specific formula is general for all instances where the WKB approximation is appropriate. It is informative to now apply this to the most straightforward example of Schwarzschild spacetime.

Perturbing around Schwarzschild:

For the particular case of spin one Schwarzschild, one sets $a \rightarrow 0$, and has the following:

$$
\mathcal{V}_{S c h, 1}(r)=\left(1-\frac{2 m}{r}\right) \frac{\ell(\ell+1)}{r^{2}}, \quad T(r)=1-\frac{2 m}{r}, \quad r_{\sigma}=3 m .
$$

Then the relevant quantities necessary to substitute into Equation (63) are:

$$
\begin{aligned}
\mathcal{V}_{S c h ., 1}^{\prime \prime}\left(r_{\sigma}\right) & =-\frac{2 \ell(\ell+1)}{81 m^{4}}, \quad \mathcal{V}_{\text {Sch., },}^{\prime \prime \prime}\left(r_{\sigma}\right)=\frac{16 \ell(\ell+1)}{243 m^{5}}, \\
T\left(r_{\sigma}\right) & =\frac{1}{3}, \quad T^{\prime}\left(r_{\sigma}\right)=\frac{2}{9 m},
\end{aligned}
$$

and the approximate shift in the QNMs is given by

$$
\begin{aligned}
& \delta\left(\omega_{n}^{2}\right) \approx \epsilon[\delta \mathcal{V}]\left(r_{\sigma}\right) \\
& \quad+\left.\epsilon i\left(n+\frac{1}{2}\right)\left\{\frac{11 m}{\sqrt{\ell(\ell+1)}}[\delta \mathcal{V}]^{\prime}\left(r_{\sigma}\right)-\frac{3 m^{2}}{2 \sqrt{\ell(\ell+1)}}[\delta \mathcal{V}]^{\prime \prime}\left(r_{\sigma}\right)\right\}\right|_{r_{\sigma}=3 m} .
\end{aligned}
$$

\section{Conclusions}

The spin-dependent Regge-Wheeler potentials for the regular black hole with asymptotically Minkowski core were extracted and their qualitative features thoroughly analysed. Subsequently, the spin one and spin zero fundamental quasi-normal mode profiles were examined via first-order WKB approximation for the respective dominant multipole numbers. For small $a$, both scalar spin zero and spin one electromagnetic fluctuations propagating in a regular black hole with asymptotically Minkowski core spacetime were found to have shorter-lived, higher-energy signals than for their Schwarzschild counterparts. This qualitative result does not necessarily extend to the class of regular black hole spacetimes in static spherical symmetry. Finally, general analysis of perturbation of the Regge-Wheeler potential itself was performed, and a general result presented explicating the associated shift in the QNM profiles under the perturbation $\mathcal{V}(r) \rightarrow \mathcal{V}(r)+\delta \mathcal{V}(r)$ to first-order in $\epsilon$. This general result was then applied to Schwarzschild spacetime.

Future research could include performing these calculations to higher-order in WKB, using the improved version of WKB with Padé approximants, comparing the QNM profiles to those extracted using a different method to WKB (say, e.g., time domain integration), and numerical refinement of the approximations. It would also be prudent to extend the analysis to the spin two axial mode. Discovery of a candidate spacetime which is the asymptotically Minkowski analog to Kerr, on which the wave equation separates, would also be of high interest, giving an astrophysically relevant candidate spacetime which hopefully possesses a ringdown signal that LIGO/VIRGO or LISA could delineate from Kerr. Furthermore, of interest is to explore the QNMs for when the candidate spacetime is modelling a compact massive object which is something other than a black hole; i.e., when $a>2 m /$ e. 
Funding: This research was indirectly supported by the Marsden Fund, via a grant administered by the Royal Society of New Zealand.

Acknowledgments: A.M.S. was supported by a Victoria University of Wellington PhD scholarship, and was also indirectly supported by the Marsden Fund, via a grant administered by the Royal Society of New Zealand. A.M.S. would also like to thank Matt Visser for useful conversations and discussions.

Conflicts of Interest: The author declares no conflict of interest.

\section{References}

1. Vishveshwara, C.V. Scattering of Gravitational Radiation by a Schwarzschild Black-hole. Nature 1970, 227, 936-938. [CrossRef] [PubMed]

2. Zerilli, F.J. Gravitational field of a particle falling in a schwarzschild geometry analyzed in tensor harmonics. Phys. Rev. D 1970, 2, 2141-2160. [CrossRef]

3. Press, W.H. Long Wave Trains of Gravitational Waves from a Vibrating Black Hole. Astrophys. J. Lett. 1971, 170, L105-L108 [CrossRef]

4. Davis, M.; Ruffini, R.; Press, W.H.; Price, R.H. Gravitational radiation from a particle falling radially into a Schwarzschild black hole. Phys. Rev. Lett. 1971, 27, 1466-1469. [CrossRef]

5. Price, R.H. Nonspherical perturbations of relativistic gravitational collapse. I. Scalar and gravitational perturbations. Phys. Rev. D 1972, 5, 2419-2438. [CrossRef]

6. Price, R.H. Nonspherical Perturbations of Relativistic Gravitational Collapse. II. Integer-Spin, Zero-Rest-Mass Fields. Phys. Rev. D 1972, 5, 2439-2454. [CrossRef]

7. Bardeen, J.M.; Press, W.H. Radiation fields in the schwarzschild background. J. Math. Phys. 1973, 14, 7-19. [CrossRef]

8. Zerilli, F.J. Perturbation analysis for gravitational and electromagnetic radiation in a reissner-nordstroem geometry. Phys. Rev. D 1974, 9, 860-868. [CrossRef]

9. Chandrasekhar, S.; Detweiler, S.L. The quasi-normal modes of the Schwarzschild black hole. Proc. R. Soc. Lond. A 1975, 344, 441-452. [CrossRef]

10. Detweiler, S.L. Resonant oscillations of a rapidly rotating black hole. Proc. R. Soc. Lond. A 1977, 352, 381-395. [CrossRef]

11. Detweiler, S.L.; Szedenits, E. Black Holes and Gravitational Waves. II. Trajectories Plunging into a Nonrotating Hole. Astrophys. J. 1979, 231, 211-218. [CrossRef]

12. Blome, H.J.; Mashhoon, B. Quasi-normal oscillations of a Schwarzschild black hole. Phys. Lett. A 1984, 100, 231. [CrossRef]

13. Ferrari, V.; Mashhoon, B. New approach to the quasinormal modes of a black hole. Phys. Rev. D 1984, 30, 295-304. [CrossRef]

14. Bachelot, A.; Motet-Bachelot, A. The Resonances of a Schwarzschild black hole. Ann. Inst. H. Poincare Phys. Theor. 1993, 59, 3-68. (In French)

15. Fiziev, P.P. Exact Solutions of Regge-Wheeler Equation and Quasi-Normal Modes of Compact Objects. Class. Quant. Grav. 2006, 23, 2447. [CrossRef]

16. Konoplya, R.A.; Zhidenko, A. Quasinormal modes of black holes: From astrophysics to string theory. Rev. Mod. Phys. 2011, 83, 793-836. [CrossRef]

17. Bronnikov, K.A.; Konoplya, R.A.; Zhidenko, A. Instabilities of wormholes and regular black holes supported by a phantom scalar field. Phys. Rev. D 2012, 86, 024028. [CrossRef]

18. Flachi, A.; Lemos, J.P.S. Quasinormal modes of regular black holes. Phys. Rev. D 2013, 87, 024034. [CrossRef]

19. Fernando, S.; Correa, J. Quasinormal Modes of Bardeen Black Hole: Scalar Perturbations. Phys. Rev. D 2012, 86, 064039. [CrossRef]

20. Ulhoa, S.C. On Quasinormal Modes for Gravitational Perturbations of Bardeen Black Hole. Braz. J. Phys. 2014, 44, 380-384. [CrossRef]

21. Toshmatov, B.; Abdujabbarov, A.; Stuchlík, Z.; Ahmedov, B. Quasinormal modes of test fields around regular black holes. Phys. Rev. D 2015, 91, 083008. [CrossRef]

22. Aneesh, S.; Bose, S.; Kar, S. Gravitational waves from quasinormal modes of a class of Lorentzian wormholes. Phys. Rev. D 2018, 97, 124004. [CrossRef]

23. Santos, E.C.; Fabris, J.C.; de Freitas Pacheco, J.A. Quasi-normal modes of black holes and naked singularities: Revisiting the WKB method. arXiv 2019, arXiv:1903.04874.

24. Aragón, A.; González, P.A.; Papantonopoulos, E.; Vásquez, Y. Anomalous decay rate of quasinormal modes in Schwarzschild-dS and Schwarzschild-AdS black holes. J. High Energy Phys. 2020, 2020, 1-27. [CrossRef]

25. Cuadros-Melgar, B.; Fontana, R.D.B.; de Oliveira, J. Analytical correspondence between shadow radius and black hole quasinormal frequencies. Phys. Lett. B 2020, 811, 135966. [CrossRef]

26. Churilova, M.S.; Stuchlik, Z. Ringing of the regular black-hole/wormhole transition. Class. Quant. Grav. 2020, 37, 075014. [CrossRef]

27. A Collection of Detection Papers from LIGO. Available online: https://www.ligo.caltech.edu/page/detection-companion-papers (accessed on 1 September 2021). 
28. A Complete List of Publications from the LIGO Scientific Collaboration and Virgo Collaboration. Available online: https: //pnp.ligo.org/ppcomm/Papers.html (accessed on 1 September 2021).

29. A List of Current Gravitational Wave Observations. Available online: https://en.wikipedia.org/wiki/List_of_gravitational_ wave_observationswikipedia.org/List_of_gravitational_wave_observations (accessed on 1 September 2021).

30. Barausse, E.; Berti, E.; Hertog, T.; Hughes, S.A.; Jetzer, P.; Pani, P.; Sotiriou, T.P.; Tamanini, N.; Witek, H.; Yagi, K.; et al. Prospects for Fundamental Physics with LISA. Gen. Relativ. Gravit. 2020, 52, 1-33. doi:10.1007/s10714-020-02691-1.

[CrossRef]

31. Parker, L.; Fulling, S.A. Quantized matter fields and the avoidance of singularities in general relativity. Phys. Rev. D 1973, 7, 2357-2374. [CrossRef]

32. Goswami, R.; Joshi, P.S.; Singh, P. Quantum evaporation of a naked singularity. Phys. Rev. Lett. 2006, 96, 031302. [CrossRef]

33. Bardeen, J.M. Non-singular general-relativistic gravitational collapse. In Proceedings of the International Conference GR5, Tbilisi, Georgia, 9-13 September 1968.

34. Hayward, S.A. Formation and evaporation of regular black holes. Phys. Rev. Lett. 2006, 96, 031103. [CrossRef]

35. Bronnikov, K.A.; Fabris, J.C. Regular phantom black holes. Phys. Rev. Lett. 2006, 96, 251101. [CrossRef]

36. Bambi, C.; Modesto, L. Rotating regular black holes. Phys. Lett. B 2013, 721, 329-334. [CrossRef]

37. Frolov, V.P. Information loss problem and a black hole model with a closed apparent horizon. J. High Energy Phys. 2014, 2014, 1-21. [CrossRef]

38. Simpson, A.; Visser, M. Black-bounce to traversable wormhole. J. Cosmol. Astropart. Phys. 2019, 2019, 042. [CrossRef]

39. Mazza, J.; Franzin, E.; Liberati, S. A novel family of rotating black hole mimickers. J. Cosmol. Astropart. Phys. 2021, $2021,082$. [CrossRef]

40. Franzin, E.; Liberati, S.; Mazza, J.; Simpson, A.; Visser, M. Charged black-bounce spacetimes. J. Cosmol. Astropart. Phys. 2021, 7, 036. [CrossRef]

41. Simpson, A.; Visser, M. Regular black holes with asymptotically Minkowski cores. Universe 2020, 6, 8. [CrossRef]

42. Berry, T.; Simpson, A.; Visser, M. Photon spheres, ISCOs, and OSCOs: Astrophysical observables for regular black holes with asymptotically Minkowski cores. Universe 2020, 7, 2. [CrossRef]

43. Culetu, H. On a regular modified Schwarzschild spacetime. arXiv 2013, arXiv:1305.5964.

44. Xiang, L.; Ling, Y.; Shen, Y.G. Singularities and the Finale of Black Hole Evaporation. Int. J. Mod. Phys. D 2013, 22, 1342016. [CrossRef]

45. Culetu, H. On a regular charged black hole with a nonlinear electric source. Int. J. Theor. Phys. 2015, 54, 2855-2863. [CrossRef]

46. Culetu, H. Nonsingular black hole with a nonlinear electric source. Int. J. Mod. Phys. D 2015, 24, 1542001. [CrossRef]

47. Culetu, H. Screening an extremal black hole with a thin shell of exotic matter. Phys. Dark Univ. 2016, 14, 1-3. [CrossRef]

48. Junior, E.L.B.; Rodrigues, M.E.; Houndjo, M.J.S. Regular black holes in $f(T)$ Gravity through a nonlinear electrodynamics source. J. Cosmol. Astropart. Phys. 2015, 10, 060. [CrossRef]

49. Rodrigues, M.E.; Junior, E.L.B.; Marques, G.T.; Zanchin, V.T. Regular black holes in $f(R)$ gravity coupled to nonlinear electrodynamics. Phys. Rev. D 2016, 94, 024062. [CrossRef]

50. Takeuchi, S. Hawking fluxes and Anomalies in Rotating Regular Black Holes with a Time-Delay. Class. Quant. Grav. 2016, 33, 225016. [CrossRef]

51. Boonserm, P.; Ngampitipan, T.; Visser, M. Regge-Wheeler equation, linear stability, and greybody factors for dirty black holes. Phys. Rev. D 2013, 88, 04150. [CrossRef]

52. Regge, T.; Wheeler, J.A. Stability of a Schwarzschild Singularity. Phys. Rev. 1957, 108, 1063. [CrossRef]

53. Boonserm, P.; Ngampitipan, T.; Simpson, A.; Visser, M. The exponential metric represents a traversable wormhole. Phys. Rev. D 2018, 98, 084048. [CrossRef]

54. Valluri, S.R.; Jeffrey, D.J.; Corless, R.M. Some applications of the Lambert W function to physics. Can. J. Phys. 2000, 78, 823. [CrossRef]

55. Valluri, S.R.; Gil, M.; Jeffrey, D.J.; Basu, S. The Lambert W function and quantum statistics. J. Math. Phys. 2009, 50, 102103. [CrossRef]

56. Boonserm, P.; Visser, M. Bounding the greybody factors for Schwarzschild black holes. Phys. Rev. D 2008, 78, 101502. [CrossRef]

57. Boonserm, P.; Visser, M. Quasi-normal frequencies: Key analytic results. J. High Energy Phys. 2011, 2011, 1-28. [CrossRef]

58. Sonoda, H. Solving renormalization group equations with the Lambert $W$ function. Phys. Rev. D 2013, 87, 085023. [CrossRef]

59. Sonoda, H. Analytic form of the effective potential in the large $N$ limit of a real scalar theory in four dimensions. arXiv 2013, arXiv:1302.6059.

60. Corless, R.; Gonnet, G.; Hare, D.; Jeffrey, D.; Knuth, D. On the Lambert W function. Adv. Comput. Math. 1996, 5, 329-359. [CrossRef]

61. Vial, A. Fall with linear drag and Wien's displacement law: Approximate solution and Lambert function. Eur. J. Phys. 2012, 33, 751. [CrossRef]

62. Stewart, S. Wien peaks and the Lambert W function. Rev. Bras. Ensino Fis. 2011, 33, 3308. [CrossRef]

63. Stewart, S. Spectral peaks and Wien's displacement law. J. Thermophys. Heat Transf. 2012, 26, 689-692. [CrossRef]

64. Visser, M. Primes and the Lambert W function. Mathematics 2018, 6, 56. [CrossRef]

65. Schutz, B.F.; Will, C.M. Black hole normal modes: A semianalytic approach. Astrophys. J. Lett. 1985, 291, L33-L36. [CrossRef] 
66. Iyer, S. Black hole normal modes: A WKB approach. 2. Schwarzschild black holes. Phys. Rev. D 1987, 35, 3632. [CrossRef]

67. Konoplya, R.A. Quasinormal behavior of the d-dimensional Schwarzschild black hole and higher order WKB approach. Phys. Rev. D 2003, 68, 024018. [CrossRef]

68. Zhidenko, A. Quasinormal modes of Schwarzschild de Sitter black holes. Class. Quant. Grav. 2004, 21, 273-280. [CrossRef]

69. Matyjasek, J.; Opala, M. Quasinormal modes of black holes. The improved semianalytic approach. Phys. Rev. D 2017, 96, 024011. [CrossRef]

70. Konoplya, R.A.; Zhidenko, A.; Zinhailo, A.F.; Higher order WKB formula for quasinormal modes and grey-body factors: Recipes for quick and accurate calculations. Class. Quant. Grav. 2019, 36, 155002. [CrossRef] 